\title{
Learning Robust Models for e-Commerce Product Search
}

\author{
Thanh V. Nguyen \\ Iowa State University \\ Nikhil Rao \\ Karthik Subbian \\ Amazon \\ Amazon \\ thanhng@iastate.edu \\ nikhilsreamazon.com \\ ksubbian@amazon . com
}

\begin{abstract}
Showing items that do not match search query intent degrades customer experience in ecommerce. These mismatches result from counterfactual biases of the ranking algorithms toward noisy behavioral signals such as clicks and purchases in the search logs. Mitigating the problem requires a large labeled dataset, which is expensive and time-consuming to obtain. In this paper, we develop a deep, endto-end model that learns to effectively classify mismatches and to generate hard mismatched examples to improve the classifier. We train the model end-to-end by introducing a latent variable into the cross-entropy loss that alternates between using the real and generated samples. This not only makes the classifier more robust but also boosts the overall ranking performance. Our model achieves a relative gain compared to baselines by over $26 \%$ in F-score, and over $17 \%$ in Area Under PR curve. On live search traffic, our model gains significant improvement in multiple countries.
\end{abstract}

\section{Introduction}

Deep learning models have shown excellent performance in the natural language domain, and this success has inspired practitioners to adapt these models to information retrieval tasks (Mitra et al., 2017; Huang et al., 2013). However, deep learning has not succeeded in these tasks due to the lack of massive labeled datasets (Dehghani et al., 2017). Another reason is that word-based representations (Mikolov et al., 2013; Pennington et al., 2014) are less useful in representing complex, informal search queries (Xiong et al., 2017) and hence provide limited understanding of the search intent. In the absence of explicit knowledge of which documents are "matched" with a search query and which are "mismatched", it is hard to learn robust deep learning models that understand the query intent and find high-quality, relevant documents.
Text-based product search is even more challenging. Simple modifications to the input query (or a product title) can completely change the search intent (or the product type, respectively). Take, for example, the query gray iphone $\mathrm{X}$ by which a user is looking for a specific phone. Slightly modified queries such as iPhone $X$ charger and case for iPhone $\mathrm{X}$ refer to different products. Therefore, it is hard for distributed representations to capture the nuances. Moreover, noisy user-behavioral signals from clicks and purchases (e.g., users purchased a phone while searching for a charger) can lead to biases in the ranking algorithms. As such, even top-ranked items may not match the search intent.

In this paper, we consider the problem of identifying query-item mismatches to enhance the ranking performance in product search. This task typically requires a large labeled dataset of matches and mismatches that we will respectively refer to as negative and positive samples. Even if we can partly afford the expensive and time-consuming labeling, acquired datasets are unbalanced and lack hard positive samples, preventing the classifier from learning a robust decision boundary. However, the above examples gray Iphone $\mathrm{X}$ and Iphone $\mathrm{X}$ charger motivate that meaningful positive samples can be artificially generated by leveraging the labeled data. In fact, we can heuristically construct a large number of negatives by observing which items are commonly purchased in response to the corresponding query. The question is that can we use such negatives to synthesize hard-to-classify positives to robustify the classifier? We illustrate the goal of the generation in Figure 1.

To this end, we develop a deep, end-to-end model that learns to identify mismatched queryitem pairs and is also capable of generating mismatched queries given an item. The task of the generator is twofold: it has to be able to generate hard-to-classify samples so that the classifier 


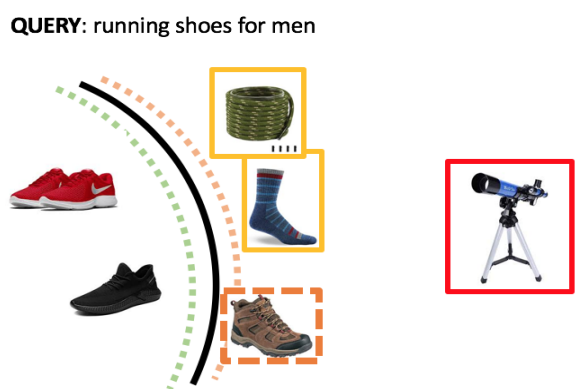

Figure 1: (Best seen in color) The query is running shoes for men. The solid black line illustrates the classification boundary, and each dotted line is a small margin around the boundary. Samples to the left of the boundary are matched. To the right are mismatches, such as socks and shoelaces (orange boxes). To the extreme right is a telescope, which is an easy-to-classify example (red box). Close to the classification boundary is a hiking shoe (orange dotted box) which is a hard-to-classify positive. We want to train a generator that can learn to generate such hard samples.

learns a more robust decision boundary; it also needs to generate realistic queries. Using matched query-item pairs allows the generator to synthesize hard-to-classify mismatches based on an efficient encoder-decoder architecture. This has a distinct advantage over generating samples from noise, as in Generative Adversarial Networks (Goodfellow et al., 2014; Wang et al., 2017) or via dithering the learned representations to make the model more robust (Miyato et al., 2018).

We include our classifier and generator in an end-to-end model. The classifier only requires continuous representations of the generated query as the second input instead of a discrete text sequence. This key property enables us to use efficient gradient-based optimization techniques and bypass reinforcement learning-based methods (Jia and Liang, 2017), which are significantly more complex, and also recently developed heuristic approaches to generate adversarial text samples (Alzantot et al., 2018). To achieve this, we modify the objective function in a way that makes the end-to-end training possible via sampling a binary latent variable, avoiding the min-max optimization for GANs (Miyato et al., 2018; Wang et al., 2017).

We perform extensive experiments on a mismatch dataset in an e-commerce company. The proposed model outperforms deep learning baselines by over $26 \%$ in F-score and $17 \%$ in relative AUPR score and performs significantly better than GBDT models, which are widely used in practice. Including the query generator helps achieve higher gains than merely dithering the vector representation of the query. We also show that the generative model can indeed generate hard-to-classify mismatches. When integrated with the ranking component of a real-world product search engine, our model outperforms the baseline methods in multiple countries on an online $\mathrm{A} / \mathrm{B}$ test evaluation.

\subsection{Problem Setup}

Let $x=(I, Q)$ denote a pair of item title and textual query and $y(I, Q)$ denote its corresponding label. $y=1$ if the pair is mismatched or $y=0$ otherwise. Assume we can obtain from search logs many matched samples, which we use to generate more positives. These samples are not human-labeled but instead inferred by considering behavioral signals such as frequent purchases.

We aim to build a deep classifier that takes two text sequences in $x_{i}=\left(I_{i}, Q_{i}\right)$ and classifies whether the pair is mismatched or not. At the same time, we want the model to generate a new sample $\left(I, Q_{\text {gen }}\right)$ with $y_{\text {gen }}=1$ given $(I, Q)$ with $y=0$. Next, we discuss our proposed model.

\section{Proposed Model: QUARTS}

We present our proposed model, namely QUARTS (QUery-based Adversarial learning for Robust Textual Search) in Figure 2. QUARTS is composed of three components: (i) an LSTM and attentionbased classifier, (ii) a variational encoder-decoder query generator (VED) and (iii) a state combiner.

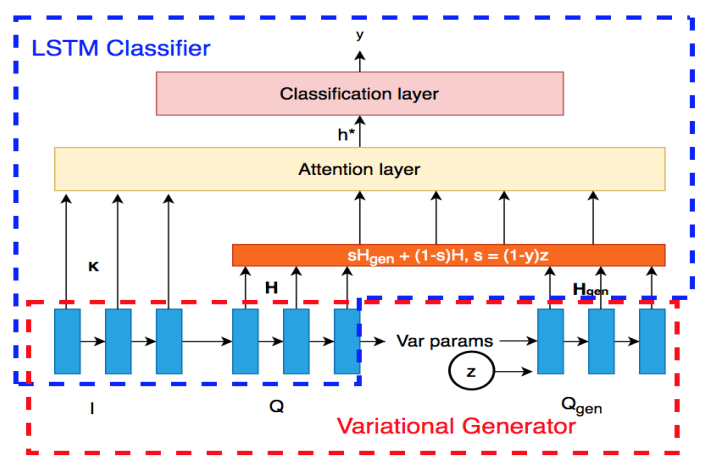

Figure 2: Our model (best seen in color). The blue dotted line encompasses the classifier. The red dotted line encompasses the generator. The orange layer in the model helps combine the outputs from the variational model and the original classifier.

Due to space constraints, we defer the details of (i) and (ii) in the appendix. The LSTM classifier (i) is adapted from the entailment model in (Rocktäschel et al., 2015), with some changes to fit the product search task (see Appendix A.1). The VED generator (ii) takes a matched pair $(I, Q)$ as input and outputs a new query $Q_{\text {gen }}$ so that the pair $\left(I, Q_{\text {gen }}\right)$ is mismatched while $Q_{\text {gen }}$ stays 
lexically similar to $Q$. As an example, if $I=$ Apple Iphone X, space gray and $Q=$ gray Iphone $\mathrm{X}$ is a matched pair, we can generate $Q_{\text {gen }}=$ Iphone $\mathrm{X}$ case given $I$. In this case, $Q_{\text {gen }}$ is similar to $Q$, but $\left(I, Q_{\text {gen }}\right)$ constitutes a product mismatch.

To have an end-to-end model, we combine the query representations computed by the classifier and the generator to form a proper input to the attention layer. We need to make sure that the modifications still allow us to efficiently backpropagate the gradients of the loss function during training. To achieve this, we add a merging layer shown by the orange box in Figure 2. This layer computes $s H_{\text {gen }}+(1-s) H, \quad s=(1-y(I, Q)) z$ where $H, H_{\text {gen }}$ are the corresponding LSTM representations of the input $Q$ and $Q_{\mathrm{gen}}$, and $z \sim$ $\operatorname{Bernoulli}(p)$ is a random binary variable that controls whether the input query $Q$ or the generated query $Q_{\text {gen }}$ is used. When $z=0$, QUARTS essentially computes the probability of mismatch.

Let us explain how the real label $y$ and the switch $z$ combine to yield the desired outputs. As $y=1$ where the sample $(I, Q)$ is a real positive, we want to leverage it to train the classifier $f_{\theta}(\cdot)$. In this case, $s=0$ and the attention layer only takes $H$ as input. When $y=0$, we can either use this sample to train the classifier or use it to generate adversarial representations $H_{\text {gen }}$. This process is controlled by $z$. When $z=1$, we use $H_{\text {gen }}$, else $H$. The value of $z$ determines whether we want to use the datapoint as-is for training, or instead use the "fake" query via the VED module.

A second consideration is how to enable efficient training on $f_{\theta}(\cdot)$ and the generator $g_{\psi}(\cdot)$. Let $x_{\text {gen }}=\left(I, Q_{\text {gen }}\right)$ be the datapoint we will use to train $f_{\theta}(\cdot)$ using the output from $g_{\psi}(\cdot)$. In this case, since $y=0, \quad z=1$, we use $z$ as a proxy "label" to train $f_{\theta}(\cdot)$. For samples $i=1,2, \ldots, N$, we sample $z_{i} \sim \operatorname{Bernoulli}(p)$ for some $p \in[0,1)$ to decide which negative samples have labels flipped. We modify the cross entropy loss as below, with $L_{\theta}$ being the weighted cross-entropy loss:

$$
\left.\frac{1}{N} \sum_{i=1}^{N}\left(1-s_{i}\right) L_{\theta}\left(x_{i}, y_{i}\right)+s_{i} L_{\theta}\left(g_{\psi}\left(x_{i}\right), z_{i}\right)\right) .
$$

Note that (1) is differentiable in $\theta, \psi$ and notably $H_{\text {gen }}$ - the generated representations of $Q_{\text {gen }}$. Since we do not use the actual generated query, we need not resort to heuristics or policy gradient-based optimization methods to minimize (1). Before train- ing QUARTS end-to-end, we pre-train the classifier and the VED on proper data. The pseudocode of the end-to-end training is shown in Algorithm 1.

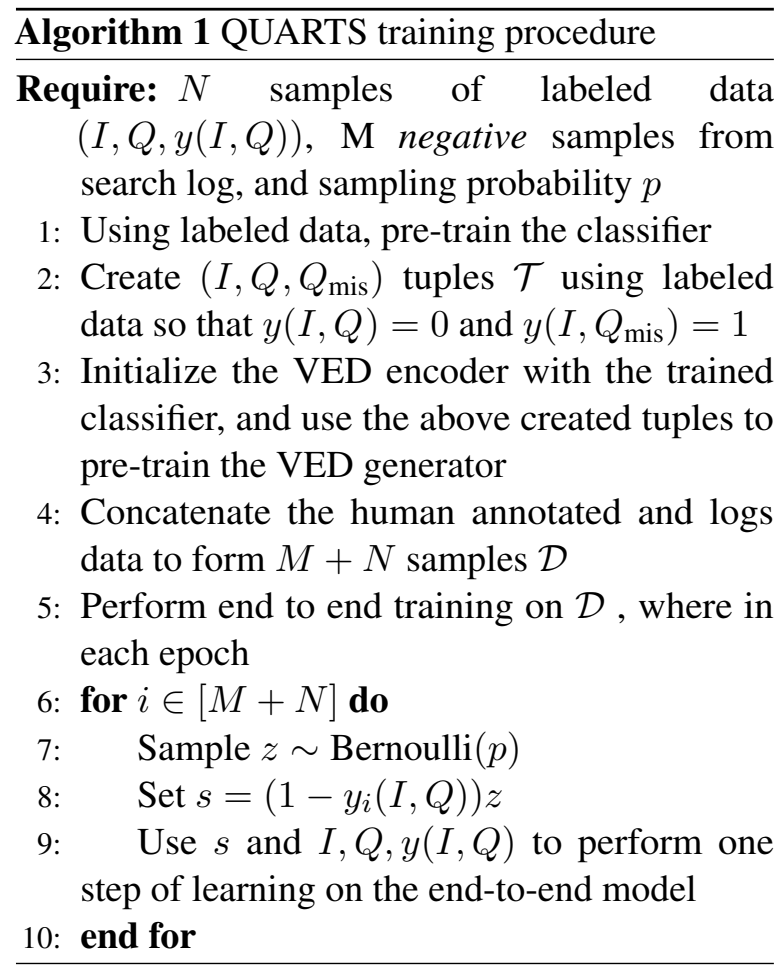

\section{Experiments and Results}

We used a human-labeled dataset of query-item pairs, obtained from an e-commerce search platform. There are in total $N=3.2 M$ pairs of which only a small fraction are mismatches. A separate test set of $\sim 100 \mathrm{~K}$ labeled pairs was used to evaluate all methods. We further have $3 M$ query-item pairs that are deemed "matched" by considering items that are purchased frequently in response to those queries from the search logs. This acts as the augmentation dataset for the QUARTS model.

\subsection{Training Details}

For all encoders and decoders, we use an LSTM with hidden size of 300 . The inputs to the encoder are 300 dimensional word embeddings trained separately for queries and item titles. The word embeddings were trained using word2 $\mathrm{vec}$ on a corpus of anonymized search engine queries, as well as item titles from the catalog. The models were trained using Adam (Kingma and Ba, 2014) and we tuned the classification part (i.e. excluding the variational decoder) on a validation dataset. We obtained the performance with initial learning rate $10^{-4}$, and learning rate decay 0.8 after 10 epochs. The dropout probability and the batch size were respectively 
0.1 and 128. Because the imbalanced nature of the labeled data, we up-weighted the positive samples. In the cross-entropy loss for classification, we set $\beta=5$.

To pretrain the VED, we used the annotated training data and generated $I, Q, Q_{\mathrm{gen}}$ tuples as explained in Section A.2. Since we are explicitly interested in training the VED to generate $Q_{\mathrm{gen}}: \quad y\left(I, Q_{\mathrm{gen}}\right)=1$ given $I, Q: y(I, Q)=0$, we consider only the annotated items that have both positive and negatively annotated queries, and generate the tuples. The previously pretrained encoder was fixed, and only the decoder was trained using Adam with an initial learning rate of $10^{-3}$. We finally merged the LSTM encoder for query and item, the VED decoder for query with the other layers described in the previous sections to train the model end to end.

The classifier $f_{\theta}(\cdot)$ is pretrained on the human annotated data. For the end-to-end model, we use the pretrained classifier and generator, modify the loss function as in (1), and further append the dataset with $M=3 M M$ well matched items from anonymized user logs, where we assume items that were purchased in response to a query are "matched" $(y(I, Q)=0)$.

\subsection{Metrics and Baselines}

We evaluated our models using Area under the Precision-Recall curve (APR), and the F1-score at the best operating point, all evaluated on the test set. To evaluate the generation task, we used BLEU scores. In addition, we had human annotators to judge generated item-query pairs. These annotators were trained to identify whether a generated pair is a match or a mismatch.

We used a GBDT model as a baseline. We used user-item features for this model similarly to traditional ranking and relevance models. We also applied a DSSM-style model (namely DSSM) where query and item word embeddings were concatenated as input to a stack of dense layers. We also used the BERT (Devlin et al., 2018) embeddings for the query and item title sequences and passed them through the aforementioned model. A final baseline we evaluated against was the MatchPyramid (Pang et al., 2016), which has shown to outperform several baselines for matching and question-answering tasks. All hyperparameters were chosen via a simple grid search on a validation set. All the results are reported on the test set.

\subsection{QUARTS Performance}

The classification results of all considered models are shown in Table 1. We also compare our model trained on the original training data and one augmented by naively adding the $3 M$ matched pairs. For confidentiality reasons, we report the performance relative to some baseline. We see from Table 1 that purely augmenting the training data with the matched samples does not improve but worsens the base classifier. Table 2 shows the performance of the QUARTS compared with MatchPyramid models and the DSSM model initialized with pretrained BERT embeddings. The end-to-end QUARTS model beats the BERT DSSM baseline by over $17 \%$ in APR, and over $26 \%$ in F-score.

\begin{tabular}{lll}
\hline Model & APR & F-score \\
\hline GBDT & baseline & baseline \\
DSSM & $+26.16 \%$ & $+28.86 \%$ \\
DSSM + BERT & $+33.71 \%$ & $+37.56 \%$ \\
MatchPyramid & $+44.95 \%$ & $+40.09 \%$ \\
QUARTS Classifier & $+52.06 \%$ & $+55.21 \%$ \\
QUARTS Classifier + Augment & $+50.9 \%$ & $+51.5 \%$ \\
QUARTS end-to-end & $+56.65 \%$ & $+62.43 \%$ \\
\hline
\end{tabular}

Table 1: The classification performance of our model on average precision and F-score, compared with baselines. The performance is relative to a GBDT model.

\begin{tabular}{lll}
\hline Model & APR & F-score \\
\hline DSSM + BERT & baseline & baseline \\
MatchPyramid & $+8.4 \%$ & $+16.44 \%$ \\
QUARTS Classifier & $+13.72 \%$ & $+20.85 \%$ \\
QUARTS end-to-end & $+17.15 \%$ & $+26.06 \%$ \\
\hline
\end{tabular}

Table 2: Comparison with other deep learning baselines.

To validate the effectiveness of QUARTS in improving the ranking performance for the search task, we performed an A/B test on live search traffic in two countries, to account for varying traffic patterns. Compared to the existing baselines, the QUARTS model yielded a $12.2 \%$ and $5.75 \%$ increase in online metrics for the two countries respectively, which are significant given the task.

\subsection{VED Results}

We used a held-out $10 \%$ of the $\left(I, Q, Q_{\text {gen }}\right)$ data to evaluate the VED generator. In order to make a fair evaluation, we ensured that the items that appeared in training set were not in the validation set. The validation BLEU scores are shown in Table 4. BLEU scores do not indicate whether or not a generated queries is a "realistic" modification of the original query. Therefore, we also had 2500 generated pairs annotated by human experts who were specifically trained to decide if a query-item 


\begin{tabular}{lll}
\hline Item title $(I)$ & Query $(Q)$ & Generated query $\left(Q_{\text {gen }}\right)$ \\
\hline ESR iPhone 8/7 screen protector tempered glass... & iPhone 8 curved screen protector & iPhone 8 plus cases \\
JETech case for iPad Pro 12.9 inch & ipad pro 12.9 speck shell & iPad pro 12.9 \\
Mounting dream full motion wall mounts bracket & lg oled tv mount & 55 inch flat screen tv \\
Intel core i7-8700K desktop processor 6 cores & core i7 8700k & GTX 1080 \\
Chicco pocket snack booster seat & peg perego high chair & baby dining set \\
Comfy sheets ultra luxury 100\% Egyptian cotton sheet set & king size sheets & king size beds for sale \\
\hline
\end{tabular}

Table 3: Examples of adversarial query generations from the VED query generator. The Item and Query should be matched, while the Item and generated query should be mismatched. For readability, we have bolded words in the query and generated query to show how the VED changes the product type intent in the generated query, while still being similar to the original query.

pair is matched or not. The accuracy $82 \%$ in Table 4 suggests that most of generated pairs are meaningful. Here, the accuracy is the fraction of the pairs that were actually labeled as mismatches

\begin{tabular}{|l|llll|l||}
\hline Model & BLEU-1 & BLEU-2 & BLEU-3 & BLEU-4 & Acc \\
\hline VED & 35.15 & 31.40 & 24.84 & 20.76 & 0.82 \\
\hline
\end{tabular}

Table 4: Validation BLEU scores of generated queries from the variational encoder-decoder generator, and misclassification accuracy as reported by humans.

We provide some qualitative results from the VED in Table 3. The generator's goal is to slightly modify the input query $Q$, so that the resultant $\left(I, Q_{\text {gen }}\right)$ sample is realistic. A source query for screen protector is mapped to a query for phone case, and a source query for $t v$ mount is mapped to one for flat screen tv.

\subsection{Word-by-Word Attention Visualization}

The goal of the word-by-word attention layer is to understand what parts of the user query and item titles are important to understand whether to match or not. Importantly, item titles are typically long, and have information such as brand, color and size. All of these facets might not be relevant for a particular user query. Figure 3 shows the performance of the word-by-word attention layer, for a matched and a mismatched pair. In both cases, we see that the correct words are attended to, helping the classifier make the distinction between a matched and a mismatched pair. Figure 4 shows another example.

\section{Conclusion and Future Work}

We developed an end-to-end model with hard to classify query generation for retrieval in ecommerce product search. We built upon ideas for textual entailment, and used a word by word attention layer to help create item representations conditioned on an input query. We trained a generator that yields representations of queries that are mismatched to a source item, while at the same time being "realistic". This allows us to address the

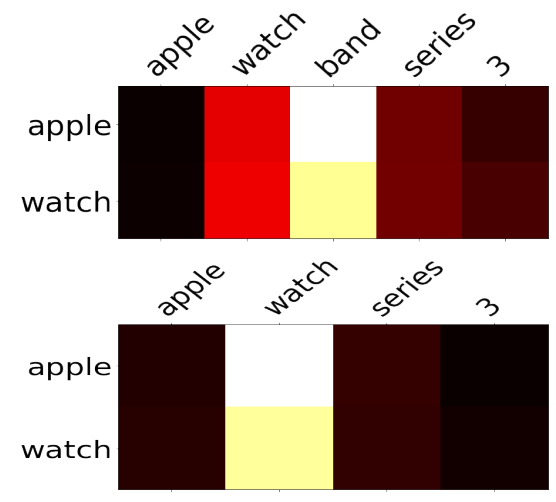

Figure 3: Word-by-word attention for a mismatched (top) and matched (bottom) query-item pair. Rows represent query words, columns represent item words, with lighter shares representing larger weights. band is attended to more on the left whereas watch is attended to more in the right

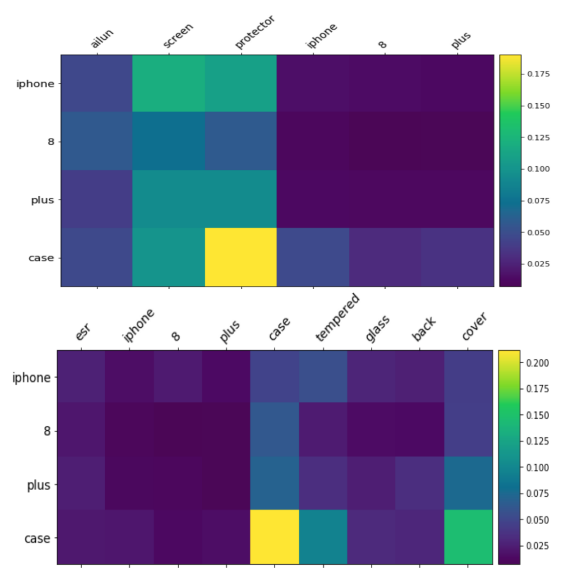

Figure 4: Word-by-word attention for a mismatched (top) and matched (bottom) query-item pair. Rows represent query words, columns represent item words, with lighter shares representing larger weights. protect or is attended to more on the left whereas case is attended to more in the right

class imbalance of our datasets, while also generating samples that help robustly train the classifier. To train the model end to end, we modified the cross-entropy loss, allowing us to avoid optimizing a minimax objective. Experiments on an offline dataset and live product search traffic showed that our method improves significantly over baselines. 


\section{References}

Moustafa Alzantot, Yash Sharma, Ahmed Elgohary, Bo-Jhang Ho, Mani Srivastava, and Kai-Wei Chang. 2018. Generating natural language adversarial examples. In Proceedings of the 2018 Conference on Empirical Methods in Natural Language Processing, pages 2890-2896, Brussels, Belgium. Association for Computational Linguistics.

Dzmitry Bahdanau, Kyunghyun Cho, and Yoshua Bengio. 2014. Neural machine translation by jointly learning to align and translate. arXiv preprint arXiv:1409.0473.

Hareesh Bahuleyan, Lili Mou, Olga Vechtomova, and Pascal Poupart. 2017. Variational attention for sequence-to-sequence models. arXiv preprint arXiv:1712.08207.

Nicholas Carlini and David Wagner. 2018. Audio adversarial examples: Targeted attacks on speech-totext. In 2018 IEEE Security and Privacy Workshops $(S P W)$, pages 1-7. IEEE.

Pin-Yu Chen, Yash Sharma, Huan Zhang, Jinfeng Yi, and Cho-Jui Hsieh. 2018. Ead: elastic-net attacks to deep neural networks via adversarial examples. In Thirty-second AAAI conference on artificial intelligence.

Minhao Cheng, Jinfeng Yi, Huan Zhang, Pin-Yu Chen, and Cho-Jui Hsieh. 2018. Seq2sick: Evaluating the robustness of sequence-to-sequence models with adversarial examples. arXiv preprint arXiv:1803.01128.

Mostafa Dehghani, Hamed Zamani, Aliaksei Severyn, Jaap Kamps, and W Bruce Croft. 2017. Neural ranking models with weak supervision. In Proceedings of the 40th International ACM SIGIR Conference on Research and Development in Information Retrieval, pages 65-74. ACM.

Jacob Devlin, Ming-Wei Chang, Kenton Lee, and Kristina Toutanova. 2018. Bert: Pre-training of deep bidirectional transformers for language understanding. arXiv preprint arXiv:1810.04805.

Javid Ebrahimi, Anyi Rao, Daniel Lowd, and Dejing Dou. 2017. Hotflip: White-box adversarial examples for text classification. arXiv preprint arXiv:1712.06751.

Ian Goodfellow, Jean Pouget-Abadie, Mehdi Mirza, Bing Xu, David Warde-Farley, Sherjil Ozair, Aaron Courville, and Yoshua Bengio. 2014. Generative adversarial nets. In Advances in neural information processing systems, pages 2672-2680.

Po-Sen Huang, Xiaodong He, Jianfeng Gao, Li Deng, Alex Acero, and Larry Heck. 2013. Learning deep structured semantic models for web search using clickthrough data. In Proceedings of the 22nd ACM international conference on Information \& Knowledge Management, pages 2333-2338. ACM.
Mohit Iyyer, John Wieting, Kevin Gimpel, and Luke Zettlemoyer. 2018. Adversarial example generation with syntactically controlled paraphrase networks. arXiv preprint arXiv:1804.06059.

Robin Jia and Percy Liang. 2017. Adversarial examples for evaluating reading comprehension systems. arXiv preprint arXiv:1707.07328.

Dongyeop Kang, Tushar Khot, Ashish Sabharwal, and Eduard Hovy. 2018. Adventure: Adversarial training for textual entailment with knowledge-guided examples. arXiv preprint arXiv:1805.04680.

Diederik P Kingma and Jimmy Ba. 2014. Adam: A method for stochastic optimization. arXiv preprint arXiv:1412.6980.

Volodymyr Kuleshov, Shantanu Thakoor, Tingfung Lau, and Stefano Ermon. 2018. Adversarial examples for natural language classification problems.

Minh-Thang Luong, Hieu Pham, and Christopher D Manning. 2015. Effective approaches to attentionbased neural machine translation. arXiv preprint arXiv:1508.04025.

Tomas Mikolov, Ilya Sutskever, Kai Chen, Greg S Corrado, and Jeff Dean. 2013. Distributed representations of words and phrases and their compositionality. In Advances in neural information processing systems, pages 3111-3119.

Bhaskar Mitra, Fernando Diaz, and Nick Craswell. 2017. Learning to match using local and distributed representations of text for web search. In Proceedings of the 26th International Conference on World Wide Web, pages 1291-1299. International World Wide Web Conferences Steering Committee.

Takeru Miyato, Toshiki Kataoka, Masanori Koyama, and Yuichi Yoshida. 2018. Spectral normalization for generative adversarial networks. arXiv preprint arXiv:1802.05957.

Liang Pang, Yanyan Lan, Jiafeng Guo, Jun Xu, Shengxian Wan, and Xueqi Cheng. 2016. Text matching as image recognition. In Thirtieth AAAI Conference on Artificial Intelligence.

Nicolas Papernot, Patrick McDaniel, Ananthram Swami, and Richard Harang. 2016. Crafting adversarial input sequences for recurrent neural networks. In MILCOM 2016-2016 IEEE Military Communications Conference, pages 49-54. IEEE.

Jeffrey Pennington, Richard Socher, and Christopher Manning. 2014. Glove: Global vectors for word representation. In Proceedings of the 2014 conference on empirical methods in natural language processing (EMNLP), pages 1532-1543.

Tim Rocktäschel, Edward Grefenstette, Karl Moritz Hermann, Tomáš Kočiskỳ, and Phil Blunsom. 2015. Reasoning about entailment with neural attention. arXiv preprint arXiv:1509.06664. 
Rico Sennrich, Barry Haddow, and Alexandra Birch. 2015. Improving neural machine translation models with monolingual data. arXiv preprint arXiv:1511.06709.

Christian Szegedy, Wojciech Zaremba, Ilya Sutskever, Joan Bruna, Dumitru Erhan, Ian Goodfellow, and Rob Fergus. 2013. Intriguing properties of neural networks. arXiv preprint arXiv:1312.6199.

Jun Wang, Lantao Yu, Weinan Zhang, Yu Gong, Yinghui Xu, Benyou Wang, Peng Zhang, and Dell Zhang. 2017. Irgan: A minimax game for unifying generative and discriminative information retrieval models. In Proceedings of the 40th International ACM SIGIR conference on Research and Development in Information Retrieval, pages 515-524. ACM.

Chenyan Xiong, Zhuyun Dai, Jamie Callan, Zhiyuan Liu, and Russell Power. 2017. End-to-end neural ad-hoc ranking with kernel pooling. In Proceedings of the 40th International ACM SIGIR conference on research and development in information retrieval, pages 55-64. ACM.

\section{A Appendices}

\section{A.1 LSTM Classifier}

We adapt our classifier from that for textual entailment in (Rocktäschel et al., 2015), but with a few key differences. Unlike standard textual entailment problems for natural language, user queries and item titles tend to follow different language patterns, with both of them being different from "natural" language. For example, queries "red nike running shoes", "running nike shoes, red" and "red running shoes nike" all refer to the same general product, despite differing in structure. On the other hand, item titles are structured, with brand, size, color, etc. all mentioned in a long sequence, which is also not how a conventional sentence is structured. To account for these differences between query strings and item titles, we separately train word embeddings using word2vec (Mikolov et al., 2013) on anonymized query logs and item titles. Thus, the same word can have two embeddings, one for the query and one for the title. The overall classifier structure is shown in Figure 5

We implement the word-by-word attention layer as follows: Let $k$ be the output dimension of the LSTMs, $K \in \mathbb{R}^{k \times m}$ and $H=\left[h_{1}, h_{2}, \ldots, h_{n}\right] \in$ $\mathbb{R}^{k \times n}$ be the LSTM output matrices for the item title and query respectively, with the $i^{\text {th }}$ column corresponding to the output of the $i^{\text {th }}$ LSTM cell. Let $m$ and $n$ denote respective lengths of title and query sequences.

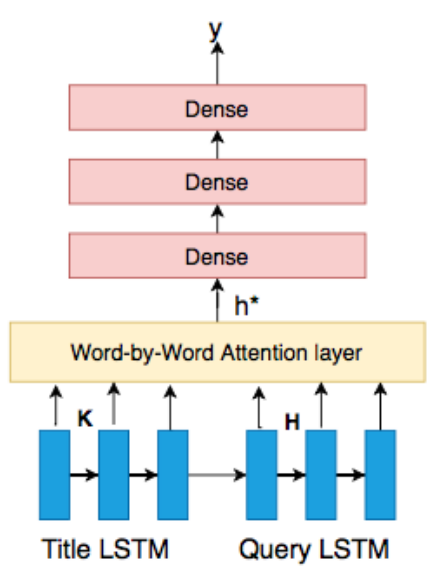

Figure 5: LSTM based classifier for a query-item pair (best seen in color). The LSTMs are fed word embeddings, separately learned for queries and titles. The word-by-word attention layer is adapted from (Rocktäschel et al., 2015), and $h^{*}$ is defined in (2)

For each word $t=1,2, \ldots, n$ in the query, we compute attention scores for every word in the title and its weighted representation $r_{t}$ at that step. The representation $r_{t-1}$ is helpful to inform the next step what the model previously paid more focus on. We use the additive attention (Bahdanau et al., 2014) here, but other alternatives can be used as well.

$$
\begin{aligned}
& M_{t}=\tanh \left(\left[K^{\top}, 1 h_{t}^{\top}, 1 r_{t-1}^{\top}\right] W_{h}\right), \\
& \alpha_{t}=\tanh \left(M_{t} w\right), \quad w \in \mathbb{R}^{k} \\
& r_{t}=K^{\top} \alpha_{t}+\tanh \left(W_{r} r_{t-1}\right) .
\end{aligned}
$$

where $W_{h} \in \mathbb{R}^{3 k \times k}$ and $W_{r} \in \mathbb{R}^{k \times k}$. The final representation for the query and title that is passed to fully-connected layers is:

$$
h^{*}=\tanh \left(W_{x}\left[r_{n}^{\top}, q_{n}^{\top},\left|r_{n}-q_{n}\right|^{\top}\right]\right),
$$

where $W_{x} \in R^{k \times 3 k}$. In the above equations, $W_{h}, w, W_{r}$ and $W_{x}$ are weight matrices to be learned, and $q_{n}$ is the output of the LSTM that encodes the query. Passing $\left|r_{n}-q_{n}\right|$ in (2) to the dense layers improves classification performance. We observe in mismatched query-item pairs that a slight word substitution or deletion often leads to mismatched items; for example, "iPhone screen protector" and "iPhone screen" or "iPad screen protector" are textually very similar, but are completely different items from a shopping point of view. Hence, we use the term $\left|r_{n}-q_{n}\right|$ in (2) to explicitly account for such word changes. Traditional sentence classification methods also pass 
$r_{n} \circ q_{n}$ to the dense layers, where $\circ$ is the hadamard product. We noticed that this did not improve the model performance, and hence choose to not use it. A desirable side effect is reduced computations. We expect that $\left|r_{n}-q_{n}\right|$ somehow captures words that are in the query but not in the title and vice versa.

Let $f_{\theta}(\cdot)$ denote the classifier in Figure 5. Given $N$ samples $\left\{\left(x_{i}, y_{i}\right)\right\}_{i=1}^{N}$, our objective function is a weighted cross-entropy loss:

$$
\begin{aligned}
L_{\theta}(X, y)= & \frac{1}{N} \sum_{i=1}^{N} L_{\theta}\left(x_{i}, y_{i}\right) \\
= & \frac{1}{N} \sum_{i} \beta y_{i} \log \left(f_{\theta}\left(x_{i}\right)\right) \\
& +\left(1-y_{i}\right) \log \left(1-f_{\theta}\left(x_{i}\right)\right),
\end{aligned}
$$

where $\beta$ adjusts the weight on the positive samples. We set $\beta>1$ to account for the fact that the number of positive samples (i.e. mismatched) is much larger than negative samples in our datasets.

\section{A.2 Variational Query Generator}

For the applications we are interested in, the training datasets are highly unbalanced, as a reasonable search engine will have far more matched queryitem examples than mismatched. We thus need ways to account for this class imbalance. Generating trivially mismatched examples is easy: we can randomly sample an item from the entire cata$\log$ for a given query. But these will be examples that are easy-to-classify for $f_{\theta}(\cdot)$, and are hence uninformative. Here we aim to train a model that can generate hard-to-classify mismatched examples, which tend to occur due to the query and product title being lexically similar. Specifically, we want to generate mismatched query-item examples that have a realistic chance of appearing in the search results for said query.

We train a Variational Encoder-Decoder (VED) model to this end. The model takes as input a matched pair $(I, Q)$, and outputs a new query $Q_{\text {gen }}$ so that the pair $\left(I, Q_{\text {gen }}\right)$ is mismatched, but being lexically similar to $Q$. As an example, if $I=$ puma running shoe, size 11, black and $Q=$ running shoes for men, we can generate $Q_{\text {gen }}=$ insoles for running shoes. In this case, $Q_{\text {gen }}$ is similar to $Q$ in that the item is somewhat related to $Q_{\text {gen }}$, and there's a chance that $I$ may be matched to $Q_{\text {gen }}$ due to keyword stuffing by sellers, or poor semantic matching. On the other hand, another mismatched query $Q_{\text {gen }}=$ pizza cutter is not a good candidate to generate, since it's highly unlikely that a reasonable search engine will show shoes for a query about pizza cutters.

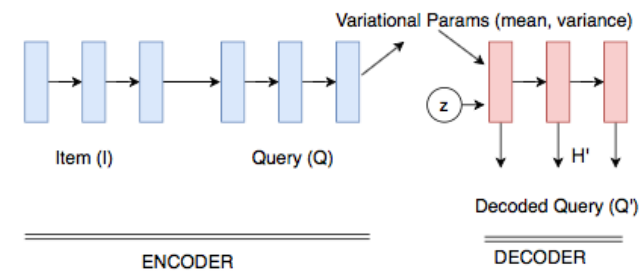

Figure 6: Variational encoder-decoder query generator (best seen in color). The encoder is reused from the classifier in the previous section. The decoder is an LSTM with attention (Luong et al., 2015). $Q_{\text {gen }}$ is a generated query via beam search.

To train the model, we make use of an labeled $\{(I, Q, y)\}$ dataset and create a new one as follows: we consider only those items $I$, for which there exist both matched and mismatched queries, and construct samples $\left(I, Q, Q_{\mathrm{mis}}\right)$ so that $y(I, Q)=0$, and $y\left(I, Q_{\text {mis }}\right)=1$. The model is the variational sequence to sequence model proposed in (Bahuleyan et al., 2017), which we adapt to our case (Figure 6). Our architecture can reuse the titlequery encoder of the classifier in Section A.1. The variational decoder allows us to generate diverse output sequences for the same input. We equip the decoder with an attention mechanism (Luong et al., 2015) to generate $Q_{\text {gen }}$. Using an existing annotated dataset to pretrain the VED allows us to accurately warm start the end to end model described in the next section.

\begin{tabular}{|l|l|}
\hline Source & Targets \\
\hline kate spade yoga mat & $\begin{array}{l}\text { kate spade } \\
\text { kate spade sale } \\
\text { kate spade wallet }\end{array}$ \\
\hline dickies overalls striped & $\begin{array}{l}\text { dickies work pant } \\
\text { 32x32 mens dickies shorts } \\
\text { kahki overalls for women }\end{array}$ \\
\hline puppy training 101 & $\begin{array}{l}\text { dog training pad } \\
\text { dog training collar } \\
\text { puppy }\end{array}$ \\
\hline plastic stacking bins & stackable storage bins \\
& storage bins \\
& foldable storage bins \\
\hline
\end{tabular}

Table 5: Nearest neighbors by cosine similarity for a few queries. Note that the LSTM and mean-pooling method accurately represents queries based on various intents. In the first case, customers looking for Kate Spade items tend to look for more items of the same brand. In the second case, the model groups queries with similar intents together. In the last 2 cases, the model groups similar queries together. 


\begin{tabular}{|c|c|}
\hline $\begin{array}{l}\text { Source } \\
\text { target } 1 \\
\text { target } 2 \\
\text { target } 3\end{array}$ & $\begin{array}{l}\text { screen replacement for iphone } 7 \text { plus in white including all tools instruction } 2 \text { screen protectors } \\
\text { iphone } 7 \text { plus screen replacement white lcd display } 3 \mathrm{~d} \text { touch screen digitizer frame assembly white } \\
\text { iphone } 6 \text { screen replacement white p zone } 47 \text { inch lcd display touch screen digitizer frame assembly } \\
\text { for iphone } 7 \text { screen replacement lcd touch screen digitizer frame assembly full set }\end{array}$ \\
\hline $\begin{array}{l}\text { source } \\
\text { target } 1 \\
\text { target } 2 \\
\text { target } 3\end{array}$ & $\begin{array}{l}\text { imagine by rubie s dc superheroes harley quinn mallet costume } \\
\text { rubie s harley quinn mallet costume accessory } \\
\text { rubie s women s suicide squad harley quinn mallet as as shown one size } \\
\text { rubie s women s batman harley quinn inflatable mallet multi one size }\end{array}$ \\
\hline $\begin{array}{l}\text { source } \\
\text { target } 1 \\
\text { target } 2 \\
\text { target } 3\end{array}$ & $\begin{array}{l}\text { nike unisex core golf visor dark grey anthracite white one size } \\
\text { nike golf unisex legacy } 91 \text { hat white black one size } \\
\text { nike men s flex core golf shorts dark grey dark grey size } 36 \\
\text { nike golf tech visor black adjustable one size }\end{array}$ \\
\hline $\begin{array}{l}\text { source } \\
\text { target } 1 \\
\text { target } 2 \\
\text { target } 3\end{array}$ & $\begin{array}{l}\text { mercer culinary genesis } 6 \text { piece forged knife block set tempered glass block } \\
\text { dalstrong knife set block gladiator series knife set german hc steel } 8 \text { pc } \\
\text { j a henckels international } 13550005 \text { statement knife block set } 15 \text { pc light brown } \\
\text { top chef by master cutlery } 5 \text { piece chef basic knife set with nylon carrying case }\end{array}$ \\
\hline $\begin{array}{l}\text { source } \\
\text { target } 1 \\
\text { target } 2 \\
\text { target } 3\end{array}$ & $\begin{array}{l}\text { hicksholsters purple dark punisher edition wallet } \\
\text { hicksholsters kydex dark punisher edition wallet } \\
\text { silk iphone } 6 \text { s wallet case vault protective credit card grip cover wallet slayer vol } 1 \text { black onyx } \\
\text { kalmore genuine leather rfid protected slim thin pocket wallet minimalist wallet money clip light blue }\end{array}$ \\
\hline
\end{tabular}

Table 6: We show nearest neighbors by cosine similarity based on embedding for a few item titles. In each cell, the first line (bolded) represents the source, and the next 3 lines represents its three nearest neighbors. Unlike the query case, the nearest neighbors are always substitutable items.

\section{A.3 Learned LSTM Embeddings}

Next, we verify that the learned query and item embeddings from the LSTM models are informative. To compute a vector representation of a query, we mean-pool the query LSTM outputs $H$. Similarly, for an item, we mean-pool the item LSTM outputs $K$. Table 5 shows the 3 -nearest neighbors for user queries. The neighbors are computed based on the cosine similarity between the embeddings of the source and target query. We can see that, depending on the specific query, the model learns to group queries that have the same product intent, or brand (for higher-end) items.

Along the same line, Table 6 shows the 3 -nearest neighbors in terms of cosine similarity for items. Note that this case is not the same as queries, since an item by itself is meaningless. Indeed, the outcome of the word by word attention model is to achieve an item representation conditioned on the query. More specifically, the item nike running shoe by itself cannot be deemed as matched or mismatched, unless seen in the context of a user typed query. Hence, the LSTM + mean pooling output for the items tend to cluster similar (substitutable) items together. The upshot if this is, conditioned on a given query $Q$, the item embeddings for similar items will be similar, which is a desirable outcome for our use case.

\section{A.4 Related Work}

The DSSM (Huang et al., 2013) model and it's variants (Mitra et al., 2017; Xiong et al., 2017) have been commonly applied in learning to rank tasks. Such models are useful for web search, where there are several related documents and it's easier for natural language based models to distinguish between related and unrelated documents. These models do not easily carry over for product search, due to the issues alluded to in the previous section. Recently, (Kang et al., 2018) developed means to generate adversarial samples to improve entailment, via the use of additional datasets to learn "rules" to aid in sample generation. These rules do not carry over to the product search domain, nor do the assumption of existing datasets to learn such rules. To the best of our knowledge, we are the first to work on generating adversarial representations of text for the purpose of improving product relevance for e-commerce.

Adversarial example generation has been studied in the context of images (Szegedy et al., 2013; Chen et al., 2018), speech (Carlini and Wagner, 2018) and text (Cheng et al., 2018; Ebrahimi et al., 2017; Kuleshov et al., 2018; Iyyer et al., 2018; Papernot et al., 2016; Kang et al., 2018; Wang et al., 2017). In (Alzantot et al., 2018; Ebrahimi et al., 2017), the authors develop a means to perturb the sequence in order to fool an underlying classifier, and in (Iyyer et al., 2018), the authors use the concept of backtranslation (Sennrich et al., 2015). The aims in these works is to generate adversarial text samples themselves, separate from generating samples that will make the underlying classifier more robust. 